\title{
Clinical Evaluation of Platelet Concentrates Either in Plasma or in Addi- tive Solution
}

\author{
Luis Larrea*, $^{*}$, Nelly Carpio ${ }^{2}$, Cristina Arbona ${ }^{3}$, María Angeles Soler ${ }^{1}$, Javier de la Rubia ${ }^{2}$ and \\ Roberto Roig ${ }^{1}$
}

\author{
${ }^{1}$ Centro de Transfusión de la Comunidad Valenciana, Spain \\ ${ }^{2}$ Hospital Universitario La Fe. Valencia, Spain \\ ${ }^{3}$ Hospital Clínico Universitario. Valencia, Spain
}

\begin{abstract}
Background and Objectives. Platelet concentrates (PC) obtained from Buffy-Coat (BC) may be diluted in a platelet additive solution (PAS). These PCs may reduce plasma-related adverse reactions. We have tried to assess the relationship between PAS PCs and adverse reactions. Materials and Methods. During 6 months, patients treated with intensive chemotherapy, participated in a prospective study and were randomly assigned to receive, on a prophylactic basis, PCs in either plasma or PAS-2. Five iso-group BCs were pooled diluted in either plasma or PAS-2. One hour after each transfusion, corrected count increments (CCIs) were calculated, presence of hemorrhage and adverse reactions were recorded. Results. Platelet increment, 1-hour platelet count, and corrected count increments (CCI) after transfusion in both groups were similar. There were more transfusion- dependent adverse reactions in plasma group. Conclusion. Use of PAS-2 resulted in less transfusion related reactions; therefore, we recommend synthetic additive solutions to dilute PCs.
\end{abstract}

Keywords: Platelet transfusions, PAS, Adverse reactions.

\section{BACKGROUND AND OBJECTIVES}

Bleeding in a number of acute and chronic conditions that require medical and surgical treatment may be prevented and treated with platelet transfusions [1]. Prophylactic platelet concentrate (PC) transfusions in patients with severe thrombocytopenia have increased over the last years due to more frequent and more intensive radiations and chemotherapy treatments. PC may be obtained from whole blood collected into a multiple plastic bag set. The procedures for the preparation of PC from whole blood donations are the buffycoat (BC) and the platelet-rich plasma (PRP) methods. The former one is performed in Europe and the latter in North America and in several European countries. PCs obtained from $\mathrm{BC}$ may be diluted in autologous plasma or in a platelet additive solution (PAS). The use of a synthetic medium reduces the plasma content in every PC and therefore may reduce plasma-related adverse reactions. We have performed a prospective clinical trial trying to assess the relationship between PAS PCs, adverse reactions and their clinical effectiveness.

\section{MATERIALS AND METHODS}

\section{PCs Preparation}

PCs were prepared from pooled whole-blood-derived BCs. Units of whole blood $(450 \pm 50 \mathrm{~mL})$ were collected from volunteer donors in triple-bag bottom-and-top systems (Baxter. La Châtre, France) containing $63 \mathrm{~mL}$ of CPD and stored at $22^{\circ} \mathrm{C}$. Within 20 hours after collecting, the unit of whole blood was separated into its components by high-speed

*Address correspondence to this author at the Centro de Transfusión de la Comunidad Valenciana, Avda del Cid 65, 46014 Valencia, Spain; Tel: 34963868143; Fax: 34963502469; E-mail: larrea_lui@gva.es centrifugation in an Heraeus centrifuge $(6000,8000$ and 8500 ) and placed in an automated extractor system (Optipress II, Baxter) for the collection of red blood cells (RBC) and plasma, leaving the $\mathrm{BC}$ (volume, $54 \pm 2 \mathrm{~mL} ; \mathrm{Hct}, 52 \pm 3 \%$ ) in the collection bag [2,3]. To obtain PCs following the Council of Europe guidelines [4], 5 iso-group Buffy-coats were pooled diluted with either $300 \mathrm{~mL}$ of plasma or $300 \mathrm{~mL}$ of PAS-2 (T-Sol, Baxter, Lessines, Belgium) and centrifuge at low speed $[5,6]$. The resulting ratio of plasma to PAS-2 in the storage medium was approximately $3: 7$. This ratio was calculated from the content of plasma in the $\mathrm{BC}$ units and the amount of PAS-2 added. The platelet-rich supernatant was collected in a 1.3-L plastic container (PL-2410, Baxter, Healthcare, Deerfield, IL) by placing the pool in an extrusion clamp. Platelet pools were stored at $22 \pm 2^{\circ} \mathrm{C}$ on a flatbed shaker.

Depending on the time of storage, Eriksson et al. [7] divided PCs in fresh (one or two days) and stored (more than two days), we adopted this classification with slight changes; before transfusion PCs transfused the first 3 days of storage were considered fresh, while PCs transfused on day 4 or 5 were considered stored. PCs were analyzed for platelet count at each centre pre and post filtration with an automatic counter (Sysmex K800) and WBC count by means of a cytometer (FACS Calibur-Becton-Dickinson).

\section{Platelet Transfusion Protocol}

During six months, following ethical approval and after informed consent was obtained, 51 patients over the age of 18 , treated with intensive chemotherapy for hematological malignancies or with stem cell transplantation as intensification treatment for solid tumors, were called for this prospective study. Patients were excluded if they had been multitransfused (more than nine units of blood derivatives previ- 
ously received) before or if a severe hemorrhage existed. Reason for excluding multitransfused patients was in order to avoid bias on platelet recovery and on adverse reactions analysis. Eligible patients were randomly assigned to receive, on a prophylactic basis, PCs suspended in either plasma or PAS-2. Ninety-nine platelet concentrates (43 with plasma and 56 with PAS-2) were transfused. The transfusion trigger for platelets was $20 \times 10^{9}$ or less. One hour after each transfusion, corrected count increments (CCIs) were calculated, presence of hemorrhage and adverse reactions were recorded. CCI values by the following equation:

$\mathrm{CCI}=\left(\right.$ posttransfusion - pretransfusion platelet countx $\left.10^{9} / \mathrm{L}\right) \mathrm{x}$ body surface area $\left(\mathrm{m}^{2}\right)$

Number of platelets transfused $x 10^{11}$.

The study was performed at the University Hospital La $\mathrm{Fe}$ and at the University Clinic Hospital, Valencia, Spain. Medication, blood pressure and temperature before and after each transfusion were recorded. The following information was also recorded: age, sex, number of previous transfused units, diagnosis, reason for admission, pre and post filtration PCs volume, platelet count and leukocyte number, and pre filtration $\mathrm{pH}$ value at $37^{\circ}$ and $22^{\circ}$ centigrade of platelet concentrates.

Platelet filtration was made at bedside with PXL3KLE PALL filter. Transfusion-dependent adverse reactions were defined according to three categories: Febrile non-hemolytic reactions (FNHTR), allergic transfusion reactions and other reactions. FNHTR were defined as an increase in body temperature $\left(>1^{\circ} \mathrm{C}\right)$ with or without chills during and up to 4 hours after the transfusion that could not be attributed to the patient's condition, and was documented by the clinical staff. Allergic transfusion reactions were characterized as the development of urticaria or rashes with pruritus during and up to 4 hours after the transfusion. Remaining adverse effects related to transfusion were categorized as "other reactions".

A comparison was made of CCI, platelet count increment, hemorrhage after transfusion, transfusion related reactions and changes of clinical parameters such as temperature, cardiac rate, blood pressure.

\section{Statistical Analysis}

For statistical analysis, Student's t test, Mann-Whitney's $\mathrm{U}$ and Pearson $\chi^{2}$ test were applied with the use of a software program. A probability of $\mathrm{p}<0.05$ was used to reject the null hypothesis.

\section{RESULTS}

Table 1 shows patient's characteristics in the studied population. Pre and postfiltration platelet number, leukocyte number and volume in plasma group PCs were significantly higher than in PAS-2 group (Table 2). Platelet count, platelet increment and CCIs one after transfusion were similar in both groups (Table 2). We also compared post filtration leukocyte count in patients with and without reactions, but we failed to show any significant difference.

Table 3 shows the CCI data found after transfusion of fresh and stored PCs. Eighteen plasma PC transfusions and 41 PAS-2 PC transfusions were given the first 3 days of storage, and they were considered fresh. The remaining PCs were transfused on day 4 or 5 , and each being considered stored. In each platelet group 1-hour CCIs found after the transfusion of fresh and stored PCs were similar. In an effort to establish a difference among groups, we estimated the efficiency of transfusion by determining the percentage of platelet transfusions above an arbitrary CCI threshold. More than $75 \%$ of both plasma $(88.8 \%)$ and PAS-2 $(73.4 \%)$ transfusions were above the CCI threshold of 7.5. When the CCI threshold was set at $15,27 \%$ of plasma PCs transfusions and $39.2 \%$ of PAS-2 PC transfusions were above that threshold.

Table 1. Patients' Characteristics

\begin{tabular}{|c|c|c|}
\hline Solid tumor & 12 & 10 \\
\hline Acute promyelocytic leukemia & 2 & 2 \\
\hline Aplastic anemia & 1 & 1 \\
\hline Myelodysplastic syndrome & 1 & 0 \\
\hline Multiple myeloma & 1 & 0 \\
\hline Weight $(\mathrm{kg}) \pm \mathrm{SD}$ & $67 \pm 10.79$ & $71.23 \pm 10.61$ \\
\hline Pretransfusion platelet count $\left(\times 10^{9} / \mathrm{L}\right) \pm \mathrm{SD}$ & $9.94 \pm 6.25$ & $11.49 \pm 7$ \\
\hline
\end{tabular}


Table 2. Values for Patient Weight, Pretransfusion Platelet Count, and Transfusion Responses for PCs in Plasma or PAS-2*

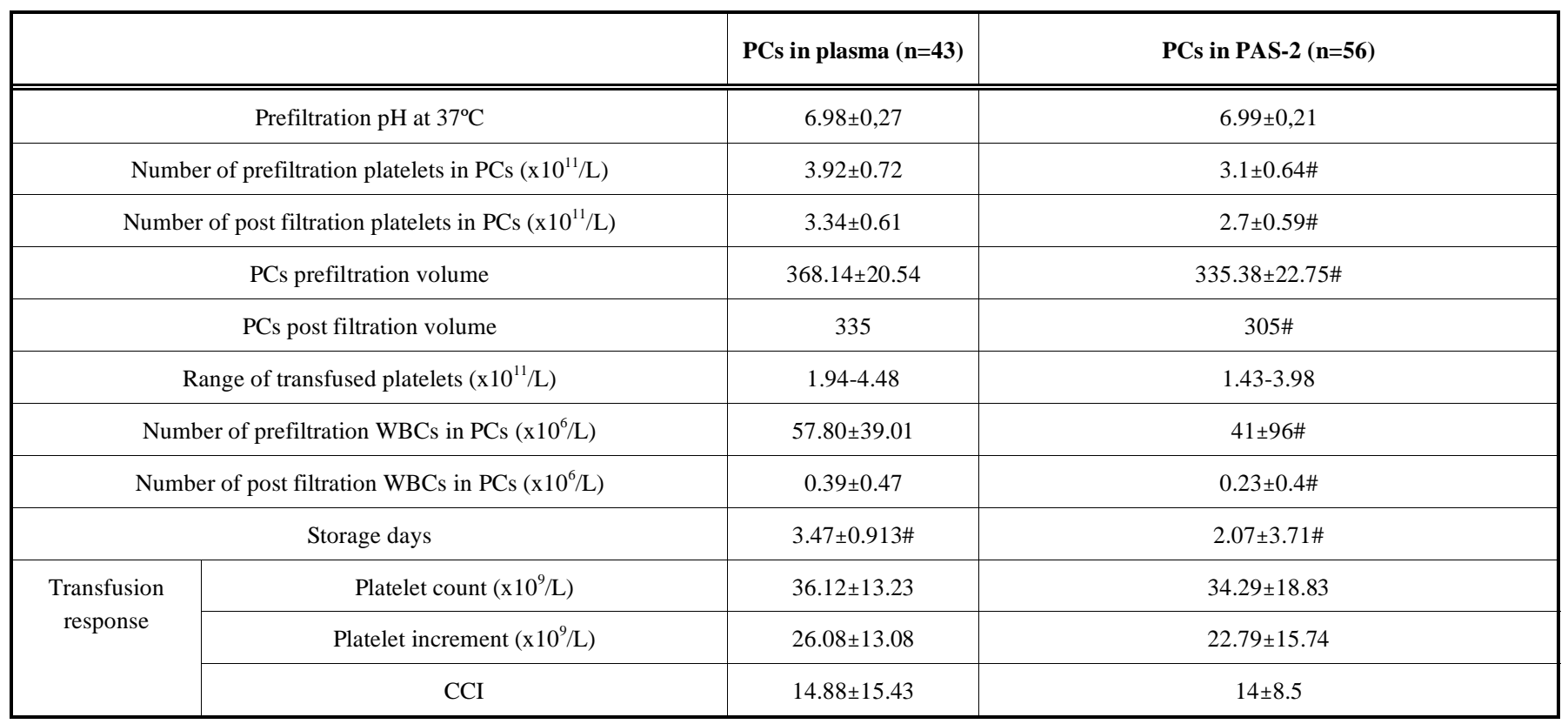

*Data are expressed as mean $\pm \mathrm{SD}$.

$\# \mathrm{p}<0.05$.

Table 3. CCIs After Transfusion of PCs in Plasma or PAS-2*

\begin{tabular}{|c|c|c|c|c|}
\hline & \multicolumn{3}{|c|}{ Component } \\
\hline & \multicolumn{2}{|c|}{ PCs in plasma } & \multicolumn{3}{c|}{ FCs in PAS-2 } & Stored \\
\hline & Fresh & Stored & $13.22 \pm 8.23$ & $14.02 \pm 7.60$ \\
\hline \hline CCI & $12.54 \pm 5.17$ & $15.57 \pm 15$ & $30 / 41(73.2 \%)$ & $9 / 10(90 \%)$ \\
\hline CCI $>7.5$ & $11 / 13(84.6 \%)$ & Transfusions above CCI threshold & $21 / 23(91.3 \%)$ & $16 / 41(39 \%)$ \\
\hline CCI $>15$ & $3 / 13(23.1 \%)$ & $7 / 23(30.4 \%)$ & $4 / 10(40 \%)$ \\
\hline
\end{tabular}

*Data are expressed as mean $\pm \mathrm{SD}$

In all cases (plasma vs. PCs in PAS-2 and fresh vs. stored) p>0.05.

We were not able to collect data about adverse reactions in three transfusion episodes from PAS-2 PC group. When we analyzed incidence of transfusion reactions we observed that in plasma group, and in PAS-2 group eight out of 43 $(18.6 \%)$ transfusions and two out of $53(3.8 \%)$ transfusions had reactions respectively, the difference was significant. Distribution of adverse reactions depending on type may be seen in Table 4. Taking only the fresh transfusions the difference of appearance of reactions between plasma and PAS2 groups kept on being significative but this difference disappeared if we analyzed only the stored PCs (Table 4).

There were scarce and mild hemorrhagic episodes after PCs transfusion without differences inter-groups ( $>0.05$ ); $6 / 43$ (14\%) in plasma group and 3/53 (5.7\%) in PAS-2 group.

No statistic relationship was found between number of previous transfusions, temperature, blood pressure and concomitant drugs with efficiency of transfusion, adverse reactions and hemorrhage.

\section{DISCUSSION}

The need for source material for plasma products and improving the quality of red cells for transfusion selected the methods for blood components in the 1970s and 1980s. The possibility to make PC from buffy-coats instead of from platelet-rich plasma has proven to be effective in increasing the national supply of plasma components and has become accepted as the normal standard procedure in the first half of the 1990s. Using this methodology we can obtain PCs with less leukocytes and spare plasma obtaining per whole blood unit more volume than with the PRP method. This saving could be increased by using PAS instead of plasma as a storage medium for PCs. After the decision to adopt PAS-2 methodology had been made we should guarantee that these PAS-2-PCs should be optimal not only taking into account in vitro results but also its clinical efficiency.

PAS displays several advantages over the use of plasma for preparation of PCs. These benefits include reduction of the number of allergic reactions $[8,9]$, while more plasma 
Table 4. Type of reactions. Incidence of Transfusion Reactions Depending on Storage

\begin{tabular}{|c|c|c|c|c|}
\hline \multirow{2}{*}{ Type of reactions } & & PCs in plasma & PCs in PAS-2 \\
\hline \hline & Allergic & 2 & \multicolumn{2}{|c|}{1} \\
\hline & FNHTR & \multicolumn{2}{|c|}{0} \\
\hline \multirow{2}{*}{$\begin{array}{c}\text { Transfusion reac- } \\
\text { tions }\end{array}$} & Discomfort & \multicolumn{2}{|c|}{ PCs in plasma\# } & PCs in PAS-2 \\
\hline & & Fresh & Stored & Fresh \\
\cline { 2 - 5 }
\end{tabular}

$\# \mathrm{p}<0.05$ comparing PCs in plasma versus PCs in PAS-2 and fresh PCs in plasma versus fresh PCs in PAS-2.

becomes available for fractioning. Due to the absence of anti-A and anti-B, ABO-incompatible transfusions are better tolerated [10], life-threatening anaphylaxis or febrile nonhemolytic transfusion reactions after transfusion of PCs is a serious clinical problem caused by the sensitizing of recipients to plasma components, such as cytokines [11]. The use of an additive solution as the major component of the platelet storage medium was first described by Rock et al. [12]. The preparation and storage of PCs in a platelet additive solution (PAS-2) have been shown to result in acceptable storage conditions [9] but require the carryover of substantial (30\%) amounts of plasma for success. Platelet additive solutions can be used as a substitute for plasma in order to recover plasma for other purposes, to avoid transfusion of large volumes of plasma to patients, to improve storage conditions, and to make possible photochemical treatment for viral inactivation of PCs [13]. Platelets remain relatively unaltered and more stable in plasma in comparison to storage in PAS-2 [14]. Currently, several platelet additive solutions for longterm platelet storage have been introduced, storing platelets in additive solution containing magnesium and potassium (PASIIIM) improves the functionality of the platelets, as measured by glycolysis, Ph, morphology, ATP and CD62 expression [15], and may allow a reduction of the amount of plasma required to be carried over to the final unit, facilitating some methods of viral inactivation and making available greater amounts of plasma for other needs [16].

In order to evaluate the clinical efficiency of PCs, CCI is to be recommended for frequent use [17]. In a prospective study a comparison of the corrected post transfusion increment was made between fresh (1-2 days) and stored (3-5 days) preparations, fresh BC-PCs gave higher increments than stored BC-PCs [7]. A major concern related to the use of PAS-2-PCs could be lost of its therapeutic properties translated into an increase of bleeding episodes; in our study, use of PAS-2 resulted in good CCI in both groups and without differences in clinical effectiveness (measured by bleeding). PAS-2 group was associated with less transfusion related reactions, we think that this reduction in adverse reactions in PAS-2-PCs may be related with the presence of fewer leukocytes or less protein content in PAS-2 platelet concentrates. Even if to be expected from the pathophysiologic mechanism (leukocytes in the case of FNHTR and plasma proteins in the case of allergic reactions), differences might be accidental due to residual plasma and leukocyte count in PAS-2-PCs, though significantly less, might be enough to cause an undesirable reaction. When we compared post filtration leukocyte count in patients with and without reactions, we failed to show any significant difference; also we could not distinguish differences in adverse reaction subgroups and type of PC, these two facts could be easily explained by the small sample size.

Platelet activity studies are still needed (under basal conditions and after activation) after platelet transfusion in both groups. Since less transfusion-dependent reactions were observed in patients who obtained PCs diluted with PAS2 instead of plasma, which could not be linked to differences in platelet counts, the study of platelet activity in both groups would greatly improve the quality of this study.

Taking into account that PAS-2-PC results in haemostatic effect of equal quality to plasma-PC and in less transfusion associated morbidity, it seems advisable to elaborate PCs using synthetic additive solutions.

\section{REFERENCES}

[1] Coluccio E. Platelet transfusion. Haematologica 2002; 87: 1121-5.

[2] Högman CF, Eriksson L, Hedlund K, et al. The bottom and top system: a new technique for blood component preparation and storage. Vox Sang 1988; 55: 211-7.

[3] Hurtado C, Bonanad S, Soler M, et al. Quality analysis of blood components obtained by automated buffy-coat layer removal with a top \& bottom system (Optipress (R)II). Haematologica 2000; 85(4): 390-5

[4] Council of Europe Publishing. Guide to the preparation use and quality assurance of blood components. $10^{\text {th }}$ edition.

[5] De Wildt-Eggen J, Schrijver JG, Smid W, et al. Platelets stored in a new-generation container: differences between plasma and platelet additive solution II. Vox Sang 1998; 75: 218-23.

[6] De Wildt-Eggen J, Bins M, van Prooijen HC. Evaluation of storage conditions of platelet concentrates prepared from pooled buffy coats. Vox Sang 1996; 70: 11-5.

[7] Eriksson L, Shanwell A, Gulliksson H, et al. Platelet concentrates in an additive solution prepared from pooled buffy-coats. In vivo studies. Vox Sang 1993; 64: 133-8.

[8] Heddle NM, Klama L, Meyer R, et al. A randomized controlled trial comparing plasma removal with white cell reduction to prevent reactions to platelets. Transfusion 1999; 39: 231-8.

[9] De Wildt-Eggen J, Nauta S, Schrijver JG, et al. Reactions and platelet increments after transfusion of platelet concentrates in plasma or in additive solution: a prospective, randomized study. Transfusion 2000; 40: 398-403.

[10] Van der Meer PF, Pietersz, R, Reesink HW. Storage of platelets in additive solution for up to 12 days with maintenance of good invitro quality. Transfusion 2004; 44: 1204-11.

[11] Schoenfeld H, Muhm M, Doepfmer U, et al. Platelet Activity in Washed Platelet Concentrates. Anestesia Analgesia, 2004; 99: $17-$ 20 . 
[12] Rock G, Swenson SD, Adams GA. Platelet storage in a plasma-free medium. Transfusion 1985; 25(6): 551-6.

[13] Gullikson H. Platelet storage media. Transfusion apheresis Science 2001; 24: 241-244.

[14] Wagner T, Vetter A, Dimovic N, et al. Ultrastructural changes and activation differences in platelet concentrates stored in plasma and additive solution. Transfusion 2002; 42: 719-727.

[15] de Wildt-Eggen J, Schriver JG, Bins M, et al. Storage of platelets in additive solutions: effects of magnesium and/or potassium. Transfusion 2002; 42: 76-80.
[16] Gullikson H, AuBuchon JP, Vesterinen M, et al. the biomedical Excellence for safer transfusion working party of the international society of blood transfusion. Storage of platelets in additive solutions: a pilot in vitro study of the effects of potassium and magnesium. Vox Sanguinis 2002; 82: 131-136.

[17] Hogman CF. Aspects of platelet storage. Transf Sci 1994; 15: 3451-355. 